\title{
Supply Chain of Relief Materials using Blockchain
}

\author{
Jeet Bhatelia $^{1, *}$, Sagar Pandita ${ }^{1}$, Abhishek Ajmera ${ }^{1}$, Narendra Shekokar $^{2}$ and Neha Katre $^{1}$ \\ ${ }^{1}$ Department of Information Technology, Dwarkadas J. Sanghvi College of Engineering, Mumbai, India \\ ${ }^{2}$ Department of Computer Engineering, Dwarkadas J. Sanghvi College of Engineering, Mumbai, India
}

\begin{abstract}
Blockchain serves as an immutable ledger which allows transactions to take place in a decentralized manner. The proposed solution incorporates the use of Blockchain for Supply Chain Management of Relief Materials after a Disaster. The proposed solution also provides many additional features when compared to the current system like being tamper-proof, providing anonymity and accountability to the users. Our solution incorporates a box-in-box approach whilst keeping tabs via tamper-proof barcodes. Barcodes will be frequently scanned at designated locations. Users also have the facility to track the donated items online through a dedicated website. For blockchain infrastructure, we have used Hyperledger Fabric.
\end{abstract}

\section{INTRODUCTION}

Natural disasters often cause massive losses to property, people and infrastructure. The United Nations statistics show that natural disasters have cost India $\$ 80$ billion over the past two decades. And when it comes to disasters, India is the worst sufferer (in terms of death \& economical losses) because of high population density. Whenever disaster strikes, people all over the world try to help the people affected by sending donations \& supplies. People use different ways in order to donate money online, some use links provided by government \& some people use links provided by private organizations.

The problem with all these systems is that the donor does not know where his/her donated items are actually going; is it going to the disaster affected people or is it going into the pockets of corrupt officials. When the government urges people to donate money \& items, they don't trust the system because of the perceived prevalence of corruption in the hierarchical government body. Even the donations that people give via online links are not accounted for; and that makes people apprehensive towards donating items. There are many fake links circulated by miscreants which dupe people into giving them their money. People want a trusted system so that they know their donations aren't being misused. The current scenario doesn't allow for traceability and accountability of the items that goes into the system. In the proposed system, a donor can track the usage of his donated items.

To do so, the ledger-based technology can be used which will bring accountability and traceability in the picture and will allow donors to see where their items really go and be assured that their donations are going in the right place. Traceability will allow backtracking of items that are allotted the category of relief material. Adulteration of data will be prevented by the decentralized peer to peer architecture of the system with backups at every node. An interface will be available to the relief camps present in the disaster affected places to request goods.

\section{LITERATURE SURVEY}

The current system is based on the three-tier architecture. A three-tier architecture is a client-server architecture in which the user-interface, middleware, database is developed and maintained separately. [1]

These systems are based upon a central point of failure. That single point of failure makes it vulnerable to attacks involving tampering with the database.

\subsection{Industry 4.0 Fostering Construction Supply Chain Management}

This Paper discusses how Industry 4.0 will be responsible for the transformation of the manufacturing industry, but it will also have an impact on the construction industry and all the supply chains related to it. [2]

\subsection{Chainweb P.O.W Parallel-Chain Architecture for High Throughput}

PoW has problems that inhibit its ability to power a high distributed economy and is aptly criticized for its use of large amounts of electricity, and congestion fees in the form of exorbitant transaction fees. Existing Proof of Work modules are known for very low throughput, even though improvements through protocol changes have been made, none have given an ideal path towards approach the throughput levels of current payment systems. [3]

\subsection{Securify: Practical Security Analysis of Smart Contract}

Permission-less blockchains allow processing of smart contracts, providing foreign nodes to communicate without depending on third party handlers. Even though being potentially promising, a number of security related concerns have weakened the trust in managing money by smart contracts. To reduce issues like these, Securify, a security analyzer for Ethereum smart

\footnotetext{
${ }^{*}$ Corresponding author: jbhatelia@,outlook.com
} 
contracts that has high throughput, is autonomous, and able to decide if the business logic is vulnerable or not.[4]

\subsection{Blockchain technology for enhancing supply chain resilience}

This paper discusses the inner workings of blockchain technology along with its architecture. It then discusses applications of blockchain in supply chain security and the various sources of supply chain risks. It describes Blockchain enabled risk management and convergence of the blockchain with IoT. [5]

\subsection{Blockchain's roles in strengthening cybersecurity and protecting privacy}

This paper talks of the numerous security features of blockchain which give it an edge over existing systems. It talks about the various challenges in cybersecurity and the possible ways to circumvent them. [6]

\section{6 t-Closeness: Privacy Beyond k-Anonymity and I -Diversity}

Generally speaking, privacy is measured by the amount of vulnerable information about an entity. Information gain can be represented as the difference between the posterior belief and the prior belief. The originality of their approach is that they separate the information gain into two parts: from that of the whole population in the released data and that about specific individuals. [7]

\subsection{Plasma: Scalable Autonomous Smart Contracts}

Plasma is a work in progress software for execution of smart contracts which is scalable to a good amount of updates per second, enabling the blockchain to be able to represent a good amount of decentralized financial apps all around the world.

Plasma consists of two parts: Reforming all computation into a set of MapReduce functions, or do Proof-of-Stake token bonding on existing blockchains. [8]

\subsection{Ganache}

Ganache is a blockchain modelling platform for Ethereum test development that can be used to deploy smart contracts, develop dapps, and do thorough testing. [9]

\subsection{Drizzle}

Drizzle is an assortment of libraries that make writing dapp UI/UX much more convenient. The core of Drizzle is based on a Redux store, providing the use of Redux. [10]

- It makes adding middleware easier to the application.

- It takes care of syncing your business logic, transaction data and more. Response time is impressive as all is kept dynamic.

\subsection{Hyperledger Fabric}

Fabric is developed and maintained by developers all around the world. It was made keeping in mind that big organizations may be using them in future. Hyperledger
Fabric is modular in nature by default bringing about flexibility, optimizing the platform for myriad use-cases like banking, insurance, healthcare, supply chain and even digital music. Fabric supports smart contracts written in languages like Java, Go and Node.js. [11]

After analysis of literature surveys, we found that we need a system that incorporates all the above mentioned blockchain related features that enables us to add integrity, transparency, accountability to the system. Centralized systems can be easily manipulated via a single-entry point. But for Decentralized systems the same manipulation needs to be done on hundreds of machines which is practically not possible, thus making it inherently more secure than centralized single point failure systems. It truly helps the donors track their product from the time of pickup to the time it reaches the Relief camp. Blockchain features an immutable digital ledger, that is extremely safe to share with $3 \mathrm{rd}$ parties and create transactions. This also reduces fraud, and errors. The benefits we tend to receive through blockchain will save time and several other efforts. The blockchain is impenetrable in any realistic cyber-attack and hacking. There are several potential advantages that companies acquire from the blockchain technology. Increasing the public trust through the share information policy and increasing quality. An absence of malpractice and so reducing PR risk as well for the organization at work. Hence, we are proposing a system which is based on Blockchain.

\section{Proposed System Architecture}

As can be seen in the below diagram, we are utilizing the platform provided by IBM, Hyperledger. This will be the core on which the blockchain and business logic will run.

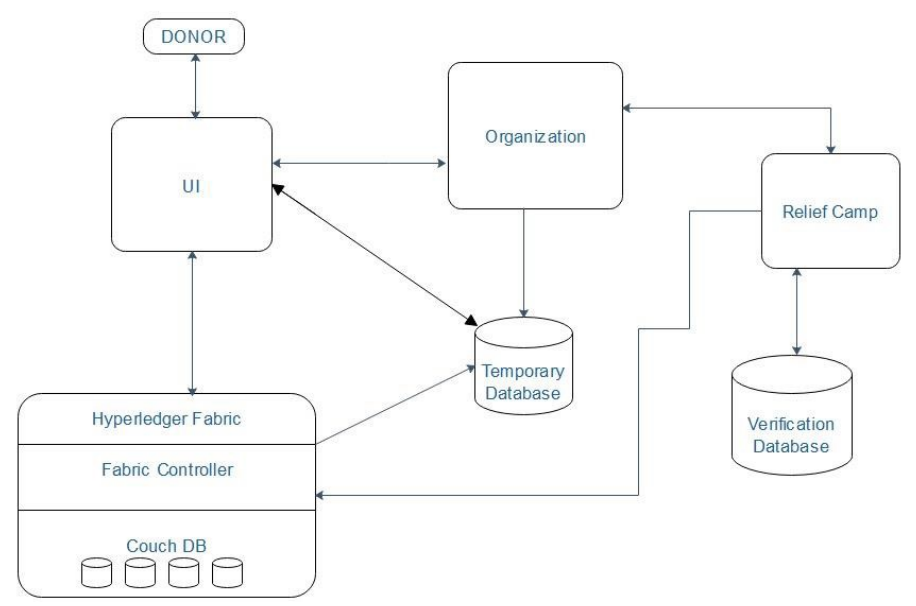

Fig. 1. Proposed System Architecture.

- The proposed system architecture consists of 4 main components and they are

- User Interface: The User Interface and the User Experience is paramount to the system as it caters to people belonging to both ends of the spectrum. Ease of use has been kept in mind while designing the UI. The learning curve needs to be very small so that uneducated and educated people can easily

\footnotetext{
${ }^{*}$ Corresponding author: ibhatelia@outlook.com
} 
use it. Cluttering on a page has been avoided methodically.

- Hyperledger Fabric Framework: This versatile platform provided by IBM will be at the core of the project. All back-end development and deployment will be taken care of using Fabric. A well-defined CLI has been provided for developers to create meaningful business logic (smart contract) to use in a project.

○ The Hyperledger Fabric consists of multiple components, a few of them include:

- Controller

- Fabric Console

- CouchDB

- We have also found ways to mitigate from the possible attacks on Blockchain. and they are:

- $51 \%$ attack: Here the evil entity tries to take over the network by owning $51 \%$ of the nodes and hence enabling them to do a hard fork and take control over the network.

○ To prevent this, we will be having a central authority that will monitor the number of nodes in the network.

- DoS Attack: It is when multiple spam nodes try to spam the honest nodes with bogus traffic and could lead to congestion of network and deny some nodes from accessing data.

$\circ$ To minimize the risk, we will change some parameters like size of block etc.

- Sybil Attacks: This is when the attacker pretends to be many people at the same time and hence changing the integrity of the data.

$\circ$ We will incorporate strict measures in the access control system to limit such cases.

- B2B Management: The system also caters to the supply chain management side of post disaster management, this communication between two businesses can be managed on the website console.

- Relief Camps: These are the well-defined spots erected after a natural disaster for disaster affected people to go to for relief material. These camps will be kept in our database while deciding where the donated items need to go to.

The flow is as follows:

- The user accesses the portal and interacts with the UI; the user then enters the disaster to which they want to make a donation to.

- Then the user is redirected to details page where he chooses the type and quantity of item he wishes to donate. He then visits a drop-off point to donate the items.

- Meanwhile the system uses an efficient way to transfer data between the website and the database. Instead of transferring the entire table containing the items entered, a 'Tag ID' is formed - a 10-bit string which encapsulates all the information about the donated item which is stored in the database. The Tag
ID can be reconstructed in a tabular form wherever required.

- At the drop-off point, the organization worker enters the donor's mobile number into the system to view the items he has entered. The worker checks the items brought by the user and matches it with the data entered.

- To ensure veracity of the items donated, the user receives a text message on his mobile phone which includes the list of items donated and an OTP which is required to conclude the transaction and only then the data is added to the Blockchain.

- Similar to above mentioned 'Tag ID', when the items are approved at the drop-off point, a Transaction ID is generated (13-bit string) which is stored in the blockchain's couchDB.

- Barcodes are stuck to each item and are read into the system via a mobile app.

- The system uses a box-in-box approach where barcodes are used to link items in boxes in a tree datastructure. The boxes in-turn are linked to the transport vehicle in a similar structure.

- The barcodes are scanned at the destination warehouse and the fabric is updated. The user can track the current location of his donated items via the website.

○ We will be using ZXing ("Zebra Crossing") barcode scanning library for decoding the barcodes.

- For creating barcodes, we used random numbers.

- Meanwhile the relief camps can request items from the organizations by viewing item statistics of each organization.

- Our website will communicate with the Node SDK with the REST APIs we created. The Node SDK can easily communicate with Hyperledger Fabric.

- We did the Hyperledger fabric development using its version 1.4 which is currently the most stable version.

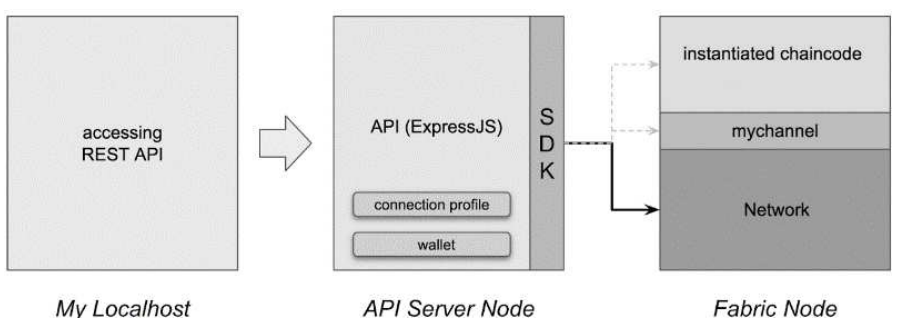

Fig. 2. Communication Architecture.

\section{Features of Proposed System}

- One of the major features of the proposed system is that the blockchain is immutable \& it has tamper detection.

- It provides anonymity to the user.

- Even the highest level of supply chain is subject to getting hacked. Blockchain on the other hand has never been hacked. The reason is that blockchain networks are secured by several computers called

* Corresponding author: ibhatelia@outlook.com 
nodes and these nodes confirm the transaction on the network.

- Other Features of the proposed system are:

- Fault Tolerance

- This means that since the whole system is distributed across multiple devices where the ledger is stored, thus increasing redundancy, and eliminating the Centralized architecture's single point of failure.

- Removal of single point of failure

- Hyperledger Fabric will use all the peers on the network, inclusive of all the organizations to replicate the ledger data. Overall, its far easier to manipulate data when its only source is a single entity. In our system it will be practically impossible for data manipulation.

- Ease of Management

- Once any data is added in the Hyperledger Network, it will automatically get replicated across all the participating organizations.

- Better Authentication

- Users who wish to donate will be able to simply login using their mobile number. An OTP will be received on their mobile, every time the user wishes to access the website, this procedure will be followed. This is a less tedious process than having passwords for each individual user who must have unique usernames. Therefore, the users do not have to remember usernames and passwords every time they want to access their website.

Table 1. Comparison between existing and proposed system.

\begin{tabular}{|l|l|l|}
\hline Donation type & $\begin{array}{l}\text { Existing } \\
\text { System } \\
\text { monetary } \\
\text { donation. }\end{array}$ & $\begin{array}{l}\text { Proposed } \\
\text { System } \\
\text { items \& } \\
\text { money }\end{array}$ \\
\hline $\begin{array}{l}\text { Donor-organization } \\
\text { consensus }\end{array}$ & Not applicable & Provided \\
\hline Item tracking & Not provided & Provided \\
\hline Disaster selection & Not provided & Provided \\
\hline $\begin{array}{l}\text { Multi-organization } \\
\text { visibility }\end{array}$ & - & Provided \\
\hline Security & $\begin{array}{l}\text { Not based on } \\
\text { Blockchain }\end{array}$ & $\begin{array}{l}\text { Based on } \\
\text { Blockchain }\end{array}$ \\
\hline
\end{tabular}

- From the above comparison table, we can see that the proposed system incorporates many additional features to the existing system like security, visibility, tracking. It follows a decentralized architecture instead of the existing client-server architecture.
- we conclude that the proposed system is able to plug most of the gaping holes in the current system through the use of Blockchain.

- This project's forefront idea is about bringing accountability. Comparing it with the current systems (Centralized) they are fast indeed but lack any sort of accountability towards the donor which our system provides.

\section{Conclusion}

In this paper, the proposed solution is customer centric and generates trust in society towards donating items for post disaster initiatives. By bringing trust, accountability and traceability in the system, it can be checked if the donated items actually reach the affected and have the donors consider it as a positive action rather than an uncertain action like we have currently wherein the third-party takes all the responsibilities without any accountability towards the donor.

\section{REFERENCES}

1. Three-Tier Architecture, https://www.techopedia.com/definition/24649/three -tier-architecture, last accessed 2019/08/21

2. P. Dallasega, IEEE Engg. Management Review, Industry 4.0 Fostering Construction Supply Chain Management: Lessons Learned From Engineer-toOrder Suppliers, 46(3), 49 (2018)

3. Chainweb, Kadena.io, 15 (to be published)

4. P. Tsankov, A. Dan, D.D. Cohen, A. Gervais, F. Bünzli, M. Vechev, arXiv:1806.01143, Securify: Practical Security Analysis of Smart Contracts, (2018)

5. H. Min, ScienceDirect, Blockchain technology for enhancing supply chain resilience, ${ }_{2}$ Volume 62 Issue 1, 35 (2019)

6. N. Kshetri, ScienceDirect, Blockchain's roles in strengthening cybersecurity and protecting privacy, Telecommunications Volume 41, Issue 10, 1027 (2017)

7. N. Li, T. Li, S. Venkatasubramanian, IEEE 23rd Int. Conf. on Data Engg., t-Closeness: Privacy Beyond $k$-Anonymity and l-Diversity (2007)

8. Plasma, working draft, Plasma.io (2017)

9. Truffle Framework - Ganache, https://ruffleframework.com/docs/ganache/overvie w, last accessed 2019/08/21

10. Truffle Framework - Drizzle, https://truffleframework.com/docs/drizzle/overview , last accessed 2019/08/21

11. Hyperledger Fabric Release Page, https://hyperledge-fabric.readthedocs.io/en/release$1.4 /$, last accessed 2019/08/21

\footnotetext{
${ }^{*}$ Corresponding author: ibhatelia@outlook.com
} 